\title{
Range-Angle-Dependent Beamforming by Frequency Diverse Array Antenna
}

\author{
Wen-Qin Wang, ${ }^{1,2}$ Huaizong Shao, ${ }^{1}$ and Jingye Cai ${ }^{1}$ \\ ${ }^{1}$ School of Communication and Information Engineering, University of Electronic Science and Technology of China, \\ Chengdu 611731, China \\ ${ }^{2}$ State Laboratory of Remote Sensing Science, Institute of Remote Sensing Applications, Chinese Academy of Sciences, \\ Beijing 100101, China \\ Correspondence should be addressed to Wen-Qin Wang, wqwang@uestc.edu.cn
}

Received 25 February 2012; Accepted 13 June 2012

Academic Editor: Marco Antonio Panduro-Mendoza

Copyright ( $) 2012$ Wen-Qin Wang et al. This is an open access article distributed under the Creative Commons Attribution License, which permits unrestricted use, distribution, and reproduction in any medium, provided the original work is properly cited.

This paper proposes a range-angle-dependent beamforming for frequency diverse array (FDA) antenna systems. Unlike conventional phased-array antenna, the FDA antenna employs a small amount of frequency increment compared to the carrier frequency across the array elements. The use of frequency increment generates an antenna pattern that is a function of range, time and angle. The range-angle-dependent beamforming allows the FDA antenna to transmit energy over a desired range or angle. This provides a potential to suppress range-dependent clutter and interference which is not accessible for conventional phased-array systems. In this paper, a FDA radar signal model is formed and the range-angle-dependent beamforming performance is examined by analyzing the transmit/receive beampatterns and the output signal-to-interference-plus-noise ratio (SINR) performance. Extensive simulation examples and results are provided.

\section{Introduction}

Phased-array antennas have been widely used in many modern communication, radar, and navigation systems [1-3]. They offer a directional gain which is useful for detecting/ tracking weak targets in the radar look direction while suppressing strong sidelobe interferences from other directions. By controlling the linear phase progression across elements, the beam can be steered to the desired direction precisely. However, a limitation of the phased-array antenna is that the beam steering is fixed in an angle for all the ranges. This limits the system performance to mitigate nondesirable range-dependent signal sources and thus degrading its ability to discern targets of interests from background noise or interference [4]. Moreover, if we want to focus the antenna beams on the directions with different ranges, multiple antennas or a multibeam antenna must be required [5-7].

Recently, a flexible beam scanning array named frequency diverse array (FDA) was proposed in [8]. The most important difference of FDA antenna from a conventional phased-array antenna is that a small amount of frequency increment compared to the carrier frequency is used across the array elements, instead of a linear phase shift like the phased array. Note that the FDA radar discussed in this paper is different from the general multiple-input multiple-output (MIMO) radar with frequency diversity where orthogonal waveforms are employed in each antenna element [9-13]. Here, the same coherent waveform is radiated from each antenna element. The use of frequency increment in FDA generates an antenna pattern that is a function of range, time, and angle [14-17].

The FDA could be used to fulfill multiple mission requirements. It was recognized in [8] that the apparent beam steering angle can be outside of real space. This provides the system designer with an increased design freedom and subsequently additional capability. It was suggested in [18] that by exploiting the multiple degrees of freedom, including space, time, frequency, and modulation, and by partitioning and combining the multidimensional space, 
the formed FDA radar can serve multiple missions simultaneously. Two patents have been issued [19, 20], which discussed the increased degrees of freedom. In [21], the continuous beam scanning feature is examined by simulation examples. In [22], the FDA was investigated from a simulation and design perspective, along with a low-cost FDA design.

In [23-25], the application of FDA in synthetic aperture radar (SAR) was investigated. The concept was to increase the angular extent of the measure scene by exploiting the bending-beam phenomenology, in order to decrease the time of the imaging platform in the scene. However, they will reject the received signals that are not frequency-matched to the transmit signals. One FDA bistatic radar system was introduced in $[26,27]$, which could have applications in lowprobability-of-intercept (LPI) radars and limiting returns from undesirable range cells. A concept by merging the waveform diverse MIMO and FDA to increase the degrees of freedom of an antenna array was proposed in [28]. Additional study was presented in $[17,29,30]$ to exploit the range-dependent characteristics and the periodic nature of the beampatterns. Although not completely associated with FDA, the phased MIMO proposed in [31] developed a flexible antenna array.

In summary, FDA provides additional degrees of freedom to efficiently gather, and therefore potentially use, scene information. However, current research undermines potential FDA capability and much further work should be carried out. For example, the FDA apparent scan angle is not equal to the nominal scan angle, which means that precise steering depends on both the range and angle. Consequently, it is insufficient to precisely steer the beam like conventional phased-array antennas.

In this paper, we present a range-angle-dependent beamforming for FDA radar. Different from the papers published in the literature $[32,33]$, where a large frequency increment is employed to ensure the required waveform orthogonality, a small frequency increment is employed in this paper. The range-angle-dependent data model is formed and the rangeangle-dependent beamforming is presented. The system performance is examined by analyzing the transmit-receive beampattern and the output signal-to-interference-plusnoise ratio (SINR) performance.

The remaining sections are organized as follows. The FDA is briefly introduced in Section 2. The range-angledependent beamforming is presented in Section 3, along with the SINR analysis. Next, extensive numerical simulation examples and results are provided in Section 4. Finally, Section 5 concludes this paper with a short discussion of future work.

\section{FDA Radar System and Signal Model}

In conventional phased arrays, it is assumed that the waveform radiated by the array elements is identical, excluding the current amplitude and current phase. Differently, the FDA elements can be either excited with the same waveform or different types of waveforms. Consider a collocated FDA

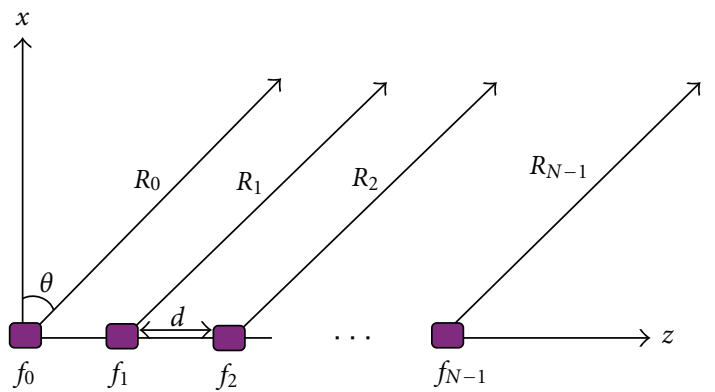

FIgure 1: The illustration of a linear frequency diverse array.

radar system with $M$ transmit elements and $N$ receive elements. For simplicity and without loss of generality, here we assume that the waveform radiated from each antenna element is identical but with a frequency increment of $\Delta f \mathrm{~Hz}$ as shown in Figure 1. That is, the radiated frequency from each element is

$$
f_{m}=f_{0}+(i-1) \cdot \Delta f, \quad m=1,2,3, \ldots, M,
$$

where $f_{0}$ is the radar operating carrier frequency.

The signal transmitted at the $m$ th element can be represented by

$$
\phi_{m}(t)=\exp \left\{j 2 \pi f_{m} t\right\} .
$$

The signal delayed to a target can then be expressed as

$$
s_{m}(t)=\exp \left\{j 2 \pi f_{m}\left(t-\frac{R_{m}}{c_{0}}\right)\right\},
$$

with

$$
R_{m} \approx R_{0}-m d \sin \theta,
$$

where $c_{0}$ is the speed of light, $d$ is the element spacing, and $\theta$ is the direction angle. The reference point is $(0,0)$ and a far-field approximation is assumed. If we steer the beam to the angle $\hat{\theta}_{0}$ and range $\hat{R}_{0}$ with the transmit beam weighting factor, we get the following:

$$
w_{T_{m}}(t)=\exp \left\{j 2 \pi f_{m}\left(\frac{\hat{R}_{0}}{c_{0}}-\frac{m d \sin \hat{\theta}_{0}}{c_{0}}\right)\right\} .
$$

This weighting function is used to achieve the maximum directional gain in the desired direction angle $\hat{\theta}_{0}$ and range $\widehat{R}_{0}$.

The transmit signal seen by the point target is given by

$$
\begin{aligned}
s_{T}(t)= & \sum_{n=0}^{M-1} \exp \left\{j 2 \pi f_{m}\left(t-\frac{R_{0}}{c_{0}}+\frac{m d \sin \theta_{0}}{c_{0}}\right)\right\} \\
& \times \exp \left\{j 2 \pi f_{m}\left(\frac{\hat{R}_{0}}{c_{0}}-\frac{m d \sin \hat{\theta}_{0}}{c_{0}}\right)\right\} \\
\approx & \exp \left\{j \Phi_{0}\right\} \frac{\sin \left(\omega_{f} M\left(t-\left(\left(R_{0}-\hat{R}_{0}\right) / c_{0}\right)\right)+\phi_{0} M \mathbb{B}\right)}{\sin \left(\omega_{f}\left(t-\left(\left(R_{0}-\hat{R}_{0}\right) / c_{0}\right)\right)+\phi_{0} \mathbb{B}\right)},
\end{aligned}
$$




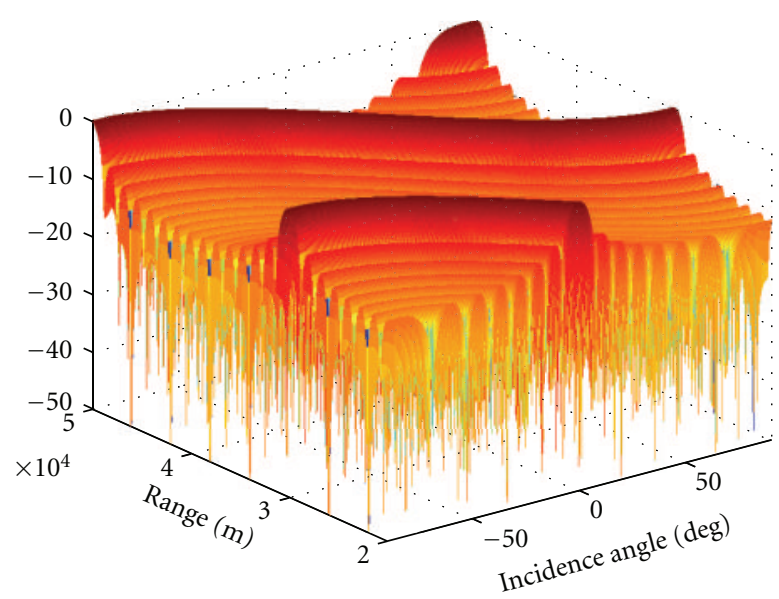

(a) Frequency diverse array radar

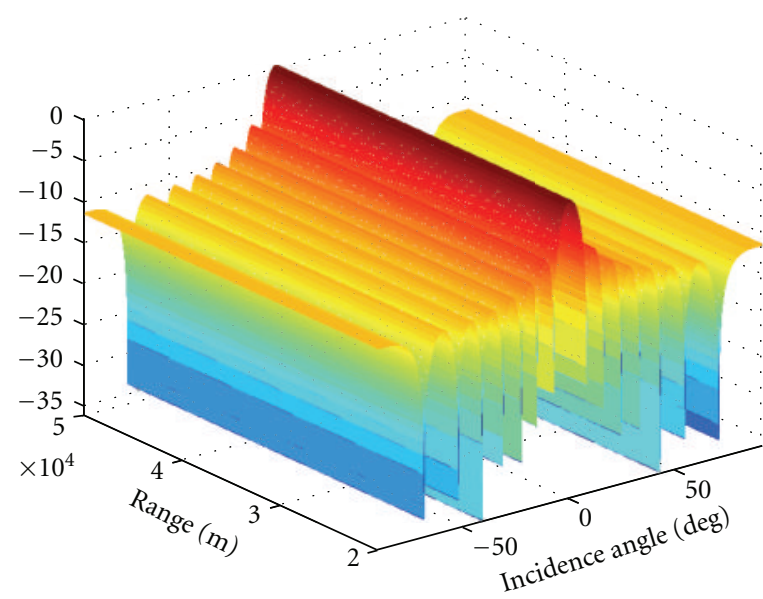

(b) Phased-array radar

FIGURE 2: Comparative beampattern between FDA and conventional phased array with $\Delta f=15 \mathrm{kHz}$.

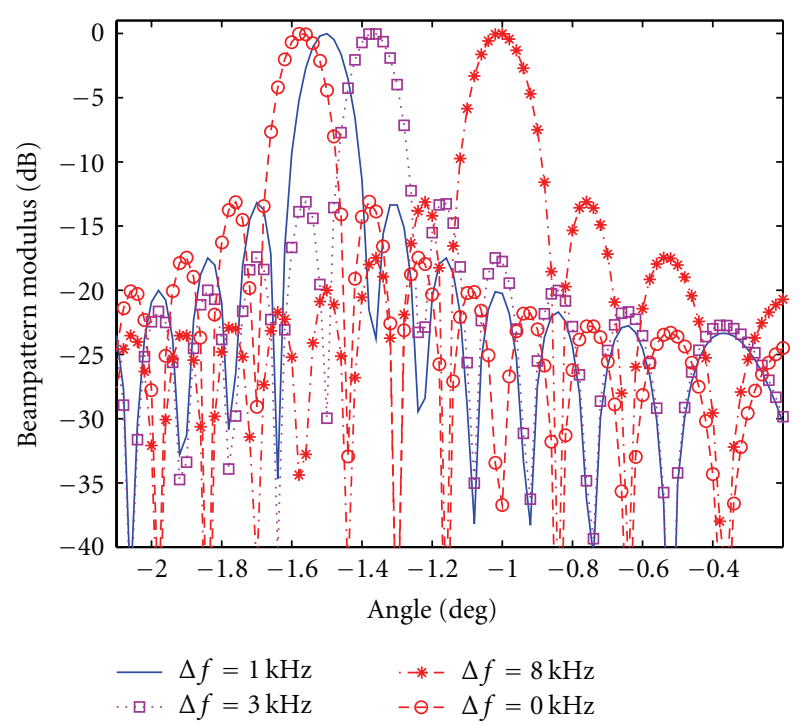

FIGURE 3: The frequency increment-dependent beam steering angle.

where $\mathbb{B}$ denotes $\left(\sin \theta_{0}-\sin \hat{\theta}_{0}\right)$.

$$
\begin{gathered}
x_{n}(t)=\sum_{n=0}^{M-1} \exp \left\{j 2 \pi f_{m}\left(t-\frac{2 R_{0}}{c_{0}}+\frac{m d \sin \theta_{0}}{c_{0}}\right)\right\} \\
\cdot \exp \left\{j 2 \pi f_{m}\left(\frac{\hat{R}_{0}}{c_{0}}-\frac{m d \sin \hat{\theta}_{0}}{c_{0}}\right)\right\} \\
\approx \sum_{m=0}^{M-1} \exp \left\{j 2 \pi f _ { m } \left(t-\frac{2 R_{0}}{c_{0}}+\frac{\hat{R}_{0}}{c_{0}}+\frac{m d \mathbb{B}}{c_{0}}\right.\right. \\
\left.\left.+\frac{n d \sin \theta_{0}}{c_{0}}\right)\right\},
\end{gathered}
$$

where

$$
\begin{gathered}
\omega_{f}=\pi \Delta f \\
\phi_{0}=\pi \frac{d}{\lambda_{0}} \\
\lambda_{0}=\frac{c_{0}}{f_{0}} .
\end{gathered}
$$

The phase term $\exp \left\{j \Phi_{0}\right\}$ is associated with the geometry of the setup.

The signal received by the $n$th element is given by (7). Similarly, using the receive beam weighting factor:

$$
w_{R_{m}}(t)=\exp \left\{j 2 \pi f_{n}\left(\frac{\hat{R}_{0}}{c_{0}}-\frac{n d \sin \hat{\theta}_{0}}{c_{0}}\right)\right\},
$$

we can get

$$
\begin{aligned}
y_{n}(t) \approx & \exp \left\{j \Phi_{1}\right\} \\
& \times \frac{\sin \left(\omega_{f} M\left(t-\left(\left(2\left(R_{0}-\hat{R}_{0}\right)\right) / c_{0}\right)\right)+2 \phi_{0} M \mathbb{B}\right)}{\sin \left(\omega_{f}\left(t-\left(\left(2\left(R_{0}-\hat{R}_{0}\right)\right) / c_{0}\right)\right)+2 \phi_{0} \mathbb{B}\right)},
\end{aligned}
$$

where $\exp \left\{j \Phi_{1}\right\}$ is the phase factors associated with the geometry of the setup. It can be noted that the periodicity of the pattern is halved in range, given that the elapsed time is now a two-way concern.

In the following, we make a summary on the FDA characteristics $\left(M=N=15, f_{0}=10 \mathrm{GHz}\right.$, and $d=\lambda / 2$ are assumed in the simulations):

(1) if the frequency offset $\Delta f$ is fixed, the beam direction will vary as a function of the range (see Figure 2), that is, it is a range-dependent beam;

(2) if the range is fixed, the beam direction will vary as a function of $\Delta f$. This means that the FDA also is a frequency-offset-dependent beam as shown in Figure 3; 
(3) if the frequency increment across the array is not applied (i.e., $\Delta f=0$ ), the corresponding FDA is just a conventional uniform linear array (ULA)-phased array. where $r$ is the distance between the first element and the observed point target. Similarly, for the second element we have

\section{Range-Angle-Dependent Beamforming}

3.1. Transmit/Receive Steering Vector. Consider a given target

$$
\psi_{2}=\frac{2 \pi f_{2}}{c_{0}}(r-d \sin \theta)=\frac{2 \pi\left(f_{1}+\Delta f\right)}{c_{0}}(r-d \sin \theta),
$$

at far field, the phase of the signal transmitted by the first element is

$$
\psi_{1}=\frac{2 \pi f_{1}}{c_{0}} r
$$

$$
\begin{aligned}
& \mathbf{a}_{t}(\theta, r)=\left[\begin{array}{llll}
1 e^{-j\left(\left(2 \pi f_{0} d \sin \theta / c_{0}\right)+\left(2 \pi \Delta f \cdot d \sin \theta / c_{0}\right)-\left(2 \pi \Delta f \cdot r / c_{0}\right)\right)} & \cdots & e^{-j\left(\left(2 \pi f_{0}(M-1) d \sin \theta / c_{0}\right)+\left(2 \pi \cdot(M-1)^{2} \Delta f \cdot d \sin \theta / c_{0}\right)-\left(2 \pi(M-1) \Delta f \cdot r / c_{0}\right)\right)}
\end{array}\right]^{T} \\
& \approx\left[\begin{array}{llll}
1 e^{-j\left(\left(2 \pi f_{0} d \sin \theta / c_{0}\right)-\left(2 \pi \Delta f \cdot r / c_{0}\right)\right)} & \cdots & e^{-j\left(\left(2 \pi f_{0}(M-1) d \sin \theta / c_{0}\right)-\left(2 \pi(M-1) \Delta f \cdot r / c_{0}\right)\right)}
\end{array}\right]^{T} \\
& =\left[\begin{array}{lllll}
1 e^{-j\left(2 \pi f_{0} d \sin \theta / c_{0}\right)} & \cdots & e^{-j\left(2 \pi f_{0}(M-1) d \sin \theta / c_{0}\right)}
\end{array}\right]^{T} \odot\left[\begin{array}{llll}
1 & e^{j\left(2 \pi \Delta f \cdot r / c_{0}\right)} & \cdots & e^{j\left(2 \pi(M-1) \Delta f \cdot r / c_{0}\right)}
\end{array}\right]^{T} \\
& =\mathbf{a}_{\Theta}(\theta) \odot \mathbf{a}_{r}(r) \text {. }
\end{aligned}
$$

The phase difference between the signals coming from the first and second elements can then be expressed as

$$
\begin{aligned}
\Delta \psi_{1} & =\psi_{2}-\psi_{1} \\
& =-\frac{2 \pi f_{0} d \sin \theta}{c_{0}}-\frac{2 \pi \cdot \Delta f \cdot d \sin \theta}{c_{0}}+\frac{2 \pi \cdot \Delta f \cdot r}{c_{0}}
\end{aligned}
$$

Similarly, the phase difference between the signals coming from the first and $(m-1)$ th elements can be expressed as

$$
\begin{aligned}
\Delta \psi_{m-1}= & -\frac{2 \pi f_{0}(m-1) d \sin \theta}{c_{0}}+\frac{2 \pi(m-1) \Delta f \cdot r}{c_{0}} \\
& -\frac{2 \pi \cdot(m-1)^{2} \Delta f \cdot d \sin \theta}{c_{0}} .
\end{aligned}
$$

The first term is simply the conventional array factor seen frequently in array theory. The second term is of importance because it shows that the radiation pattern of the array depends on the range and frequency increment. Note that the FDA is different from conventional frequency scanning arrays. Frequency-scanned arrays uses the frequency increment as a function of time for all elements, while the FDA uses the frequency increment as a function of the elements.
Taking the first element as the reference for the array, the steering vector is given by (13) with $r$ being the slant range of the first element, where

$$
\begin{gathered}
\mathbf{a}_{\Theta}(\theta)=\left[\begin{array}{lll}
1 e^{-j\left(2 \pi f_{0} d \sin \theta / c_{0}\right)} & \cdots & e^{-j\left(2 \pi f_{0}(N-1) d \sin \theta / c_{0}\right)}
\end{array}\right], \\
\mathbf{a}_{r}(r)=\left[\begin{array}{lll}
1 e^{j\left(2 \pi r \Delta f / c_{0}\right)} & \cdots & e^{j\left(2 \pi r(N-1) \Delta f / c_{0}\right)}
\end{array}\right] .
\end{gathered}
$$

Note that, since $f_{0} \gg \Delta f$ and $r \gg(m-1) d \sin \theta$, in an amplitude sense the approximation is taken by ignoring the second-phase term $2 \pi \cdot(m-1)^{2} \Delta f \cdot d \sin \theta / c_{0}$. The receive steering vector $\mathbf{a}_{r}(\theta, r)$ can be expressed in an alike manner.

3.2. Transmit/Receive Beamforming. The FDA antenna can form a directional transmit beam that points toward the look direction angle $\theta_{0}$ and range $r_{0}$. Consider $s(l)$ with $l=$ $1,2, \ldots, L$ being the number of samples of each transmitted pulse to be the baseband transmitted signal from each element. The baseband equivalent model, in a complexvalued form, of the transmitted signals from the $M$ transmit elements can be expressed as

$$
\mathbf{a}_{t}^{*}\left(\theta_{0}, r_{0}\right) s(l)
$$

where $\mathbf{a}_{t}(\theta, r)$ is just the FDA transmit steering vector containing complex-valued elements with unit amplitude and phase determined by the look angle $\theta$ and range $r$. The signal seen at a specific location with angle $\theta_{i}$ and range $r_{i}$ is a superposition of the delayed and attenuated version of 
the transmitted signals. Suppose the FDA is a narrowband system, the signal seen at that location can then be given by

$$
\mathbf{a}_{t}^{T}\left(\theta_{i}, r_{i}\right) \mathbf{a}_{t}^{*}\left(\theta_{0}, r_{0}\right) s(l)=\beta_{i} s(l)
$$

where the first $\mathbf{a}_{t}\left(\theta_{i}, r_{i}\right)$ on the left side is a propagation vector due to propagation effects, and takes the same form of the transmit steering vector. $\beta_{i}$ is the directional gain in the direction of angle $\theta_{i}$ and range $r_{i}$. It follows that the signal seen at the look angle $\theta_{0}$ and range $r_{0}$ is given by

$$
\mathbf{a}_{t}^{T}\left(\theta_{0}, r_{0}\right) \mathbf{a}_{t}^{*}\left(\theta_{0}, r_{0}\right) s(l)=\beta_{0} s(l),
$$

where $\beta_{0}=M$ with $\beta_{0}$ being the directional gain in the direction of angle $\theta_{0}$ and range $r_{0}$. Thus, we can obtain a directional gain of $M$ (the size of the transmit aperture) at the look direction. This is like the well-known property in phased-array radars which depends on only the angle, whereas for the FDA it depends on both the angle and range. Since $\left|\beta_{i}\right| \leq M$ for $\theta_{i} \neq \theta_{0}$, we have $\beta_{0} \geq \beta_{i}$. This directional gain is useful in mitigating sidelobe interference.

Suppose that a target is located at angle $\left(\theta_{0}, r_{0}\right)$ and multiple interference sources at $\left(\theta_{i}, r_{i}\right)$. The baseband equivalent of the signals at the receive array is given by

$$
\mathbf{x}(l)=\alpha_{0} M \mathbf{a}_{r}\left(\theta_{0}, r_{0}\right) s(l)+\sum_{i} \alpha_{i} \beta_{i} \mathbf{a}_{r}\left(\theta_{i}, r_{i}\right) s(l)+\mathbf{n}(l),
$$

where $\alpha_{i}$ denotes the complex amplitude of the $i$ th source, $\mathbf{a}_{r}(\theta, r)$ is an $N \times 1$ propagation vector due to the propagation delays from a source to the receive elements, and $\mathbf{n}(l)$ is the $N \times 1$ additive white Gaussian noise vector with zero mean and covariance matrix $\sigma_{n}^{2} \mathbf{I}$ with $\mathbf{I}$ being an identity matrix. The $\mathbf{a}_{r}(\theta, r)$, similarly defined as the transmit steering vector $\mathbf{a}_{t}(\theta, r)$, is the receive steering vector.

By matched filtering the received signal to the transmitted signal $s(l)$, we can get

$$
\begin{aligned}
\mathbf{y} & \triangleq \frac{\sum_{l=1}^{L} \mathbf{x}(l) s^{*}(l)}{\sum_{l=1}^{L}|s(l)|^{2}} \\
& \triangleq \alpha_{0} \mathbf{u}\left(\theta_{0}, r_{0}\right)+\sum_{i} \alpha_{i} \mathbf{u}\left(\theta_{i}, r_{i}\right)+\mathbf{n},
\end{aligned}
$$

where the virtual steering vector is

$$
\mathbf{u}\left(\theta_{i}, r_{i}\right) \triangleq \beta_{i} \cdot \mathbf{a}_{r}\left(\theta_{i}, r_{i}\right)
$$

It can be easily proved that $\mathbf{n}$ has zero mean and covariance matrix $\sigma_{n}^{2}$ I. Assuming the target/interference complex amplitudes $\alpha_{i}$ are mutually uncorrelated with zero mean and variance $\sigma_{i}^{2}$, the interference-plus-noise covariance matrix can be represented by

$$
\mathbf{R}_{i+n}=\sum_{i} \sigma_{i}^{2} \mathbf{u}\left(\theta_{i}, r_{i}\right) \mathbf{u}^{H}\left(\theta_{i}, r_{i}\right)+\sigma_{n}^{2} \mathbf{I} .
$$

We use the conventional nonadaptive beamforming, which is known to be optimal in the sense that it provides the highest possible output signal-to-noise ratio (SNR) in the background of white Gaussian noise, to derive the expression for transmit/receive beamforming pattern and output SINR performance. For this reason and due to its simplicity, the conventional nonadaptive beamformer has been widely used in antenna array systems [34]. Therefore, we use the conventional nonadaptive beamforming at both the transmit and receive arrays of the FDA radar system and derive expressions for transmit/receive beampattern and output SINR performance.

The nonadaptive beamformer weight vector for the uplink beamforming is given by

$$
\mathbf{w}_{t}=\frac{\mathbf{a}_{t}\left(\theta_{0}, r_{0}\right)}{\left\|\mathbf{a}_{t}\left(\theta_{0}, r_{0}\right)\right\|} .
$$

At the receive array, when the nonadaptive beamformer is employed, the receive beamformer weight vector is given by

$$
\mathbf{w}_{r} \triangleq \mathbf{u}\left(\theta_{0}, r_{0}\right)=M \mathbf{a}_{r}\left(\theta_{0}, r_{0}\right),
$$

where $\beta_{0}=M$ is used. The corresponding normalized FDA radar transmit/receive beampattern is

$$
\begin{aligned}
G(\theta, r) & \triangleq \frac{\left|\mathbf{w}_{r}^{H} \mathbf{u}(\theta, r)\right|^{2}}{\left|\mathbf{w}_{r}^{H} \mathbf{u}\left(\theta_{0}, r_{0}\right)\right|^{2}} \\
& =\frac{\left|\mathbf{u}^{H}\left(\theta_{0}, r_{0}\right) \mathbf{u}(\theta, r)\right|^{2}}{\left\|\mathbf{u}\left(\theta_{0}, r_{0}\right)\right\|^{4}} \\
& =\frac{\left|\left[\mathbf{a}_{\Theta}(\theta) \odot \mathbf{a}_{R}(r)\right]^{H}\left[\mathbf{a}_{\Theta}\left(\theta_{0}\right) \odot \mathbf{a}_{R}\left(r_{0}\right)\right]\right|^{2}}{\left\|\mathbf{a}_{\Theta}\left(\theta_{0}\right) \odot \mathbf{a}_{R}\left(r_{0}\right)\right\|^{4}} .
\end{aligned}
$$

It is noticed that, like the phased-array antenna, the FDA antenna has transmit coherent processing gain; however, the FDA directional gain depends on both range and angle, whereas the phased-array directional gain depends only on the range. This range-angle-dependent beam provides a potential to suppress range-dependent interferences and noise which is not available for conventional phased-array radars.

3.3. SINR Analysis. The output SINR of the FDA radar can be evaluated by

SINR

$$
\begin{aligned}
& \triangleq \frac{\sigma_{s}^{2}\left|\mathbf{w}_{r}^{H} \mathbf{u}\left(\theta_{0}, r_{0}\right)\right|^{2}}{\mathbf{w}_{r}^{H} \mathbf{R}_{i+n} \mathbf{w}_{r}} \\
& =\frac{\sigma_{s}^{2} M^{2} N^{2}}{\sum_{i} \sigma_{i}^{2}\left|\mathbf{a}_{t}^{H}\left(\theta_{0}, r_{0}\right) \mathbf{a}_{t}\left(\theta_{i}, r_{i}\right)\right|^{2}\left|\mathbf{a}_{r}^{H}\left(\theta_{0}, r_{0}\right) \mathbf{a}_{r}\left(\theta_{i}, r_{i}\right)\right|^{2}+\sigma_{n}^{2} N},
\end{aligned}
$$

where $\sigma_{s}^{2}$ is the variance of the desired target signal. If the target is observed in the background of few weak interferers which are well separated from the target, then the interference-to-noise power can be attributed to the noise term only. In this case, the SINR for the FDA radar simplifies to

$$
\operatorname{SINR} \simeq \frac{\sigma_{s}^{2} M^{2} N}{\sigma_{n}^{2}}
$$




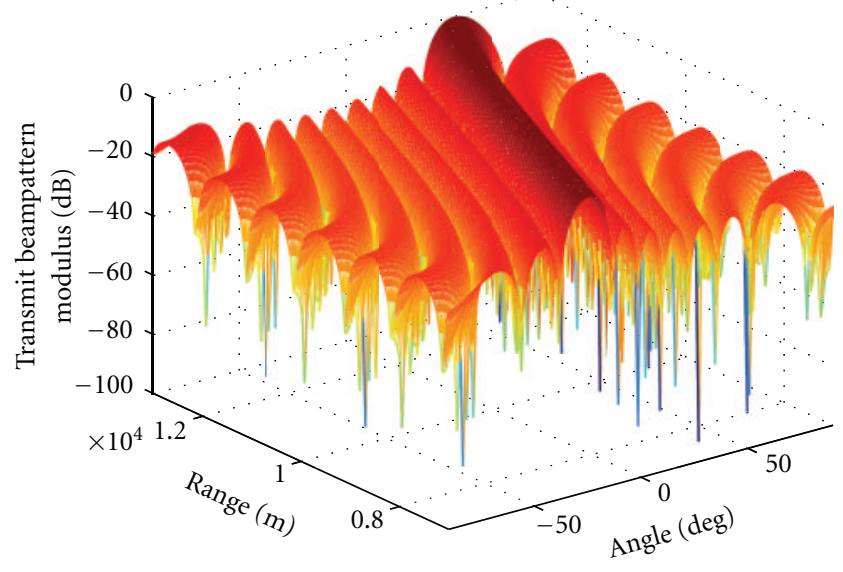

(a) Transmit beampattern

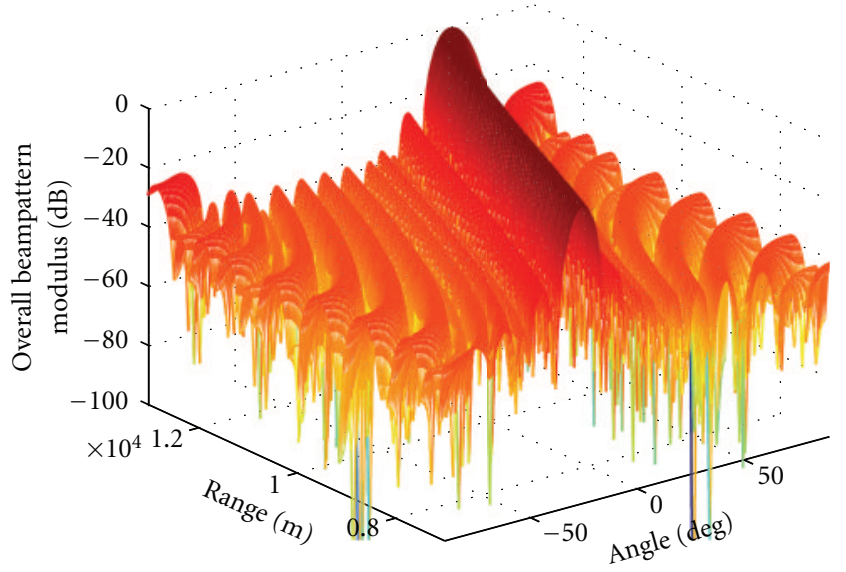

(b) Overall beampattern

FIGURE 4: The FDA beampatterns using conventional beamformer.

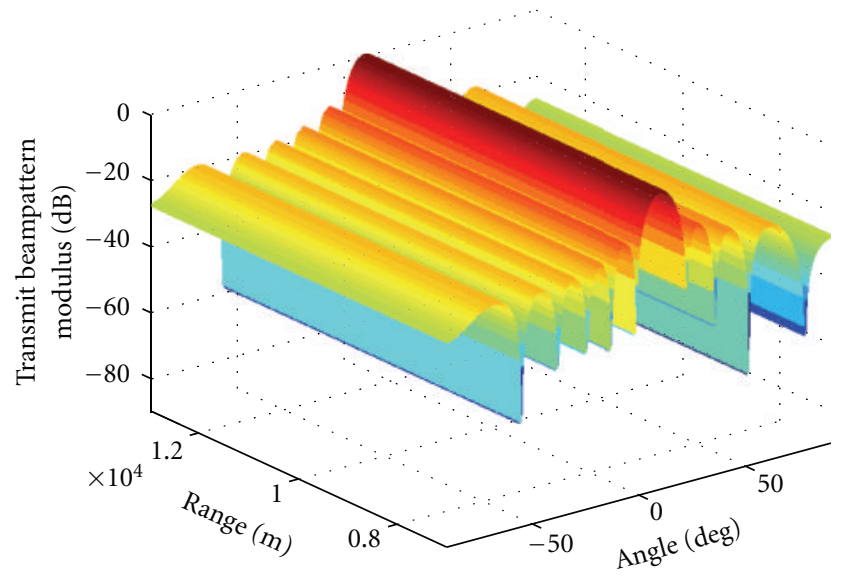

(a) Transmit beampattern

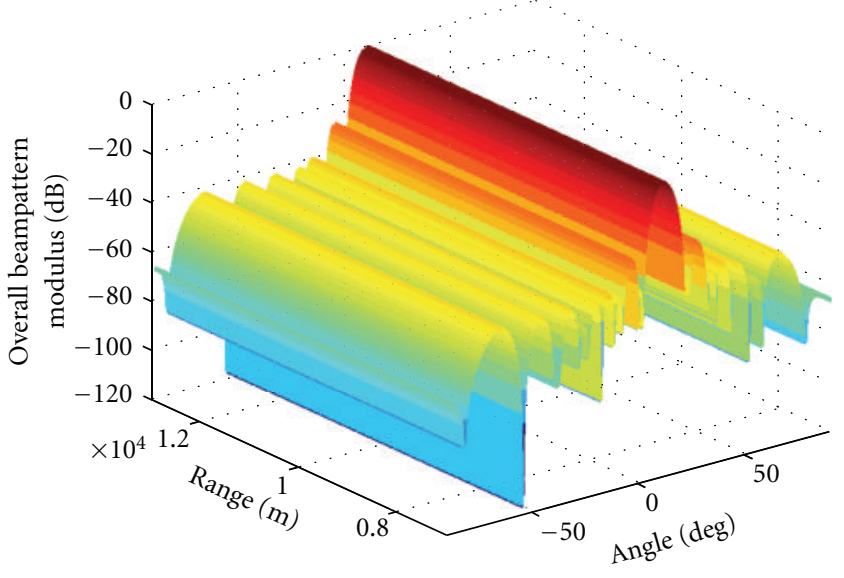

(b) Overall beampattern

FIGURE 5: Conventional phased-array beampatterns using conventional beamformer.

which means that the FDA radar is robust to background noise. In contrast, if the target is observed in the background of strong interference, then we can fairly consider the noise power to be negligible as compared to interference power. In such a case, we have

$$
\mathrm{SINR} \simeq \frac{\sigma_{s}^{2} M^{2} N^{2}}{\sum_{i} \sigma_{i}^{2}\left|\mathbf{a}_{t}^{H}\left(\theta_{0}, r_{0}\right) \mathbf{a}_{t}\left(\theta_{i}, r_{i}\right)\right|^{2}\left|\mathbf{a}_{r}^{H}\left(\theta_{0}, r_{0}\right) \mathbf{a}_{r}\left(\theta_{i}, r_{i}\right)\right|^{2}},
$$

which means that the FDA radar has a robustness against interference. By inspecting (28) and (29) we can conclude that the FDA radar has a range-angle-dependent beamforming robustness against noise and interference. This provides a capability to suppress the range-angle-dependent noise and interference.

\section{Simulation Results and Discussions}

In our simulations, we assume a ULA of $M=10$ array elements used for transmitting the baseband waveform and $N=10$ array elements at the receiving end. Both the transmitting and receiving elements are spaced half a wave length apart from each other. The additive noise is modeled as a complex Gaussian zero mean spatially and temporally white random sequence that has identical variance in the array sensor. In the following, we performed several typical simulation examples assuming the carrier frequency $f_{0}=$ $10 \mathrm{GHz}$ and $\Delta f=30 \mathrm{kHz}$.

Example 1 (one target and no interference). In the first example, one target of interest is supposed to reflect a plane wave that impinges on the array from direction of angel $\theta_{0}=$ $10^{\circ}$ and range $r=10 \mathrm{~km}$. Figures 4 and 5 show, respectively, the comparative transmit beampattern and overall transmit/receive beampattern when compared to the conventional 


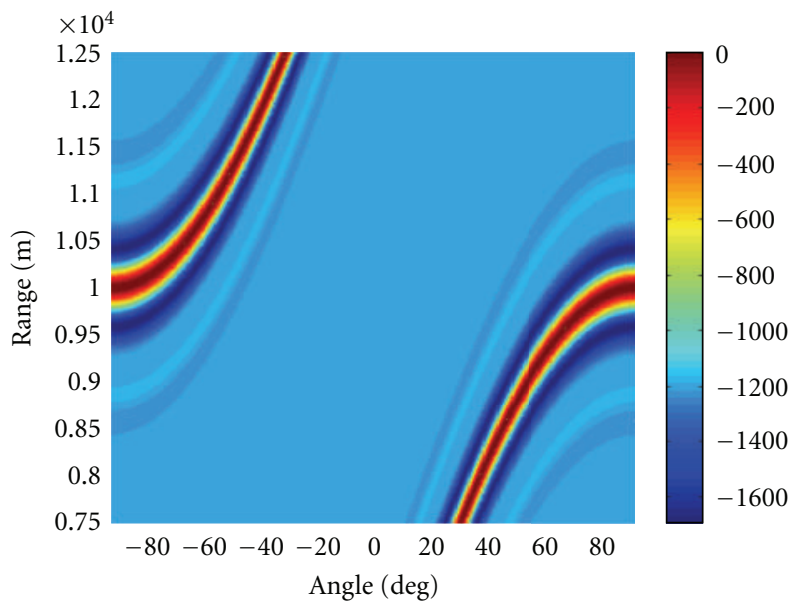

(a) FDA transmit/receive beampattern

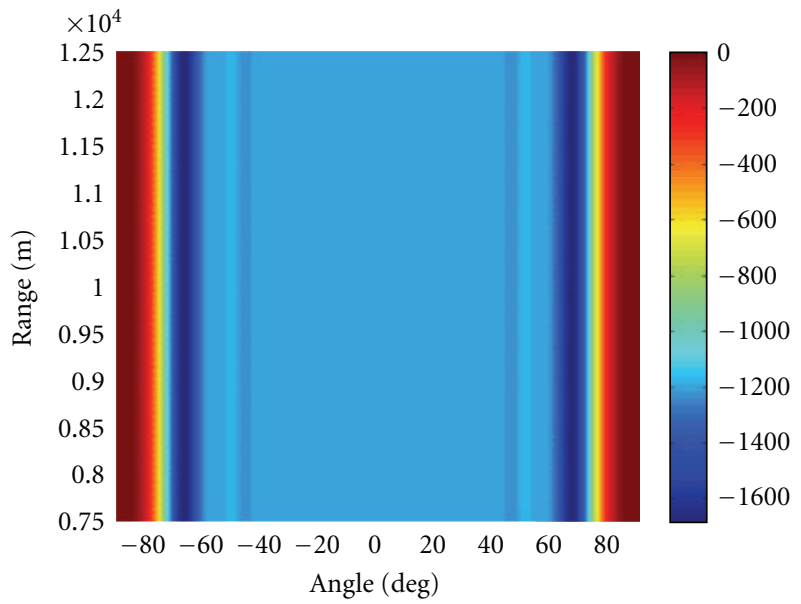

(b) Phased array transmit/receive beampattern

FIGURE 6: The overall transmit/receive beampattern when compared to a phased array.

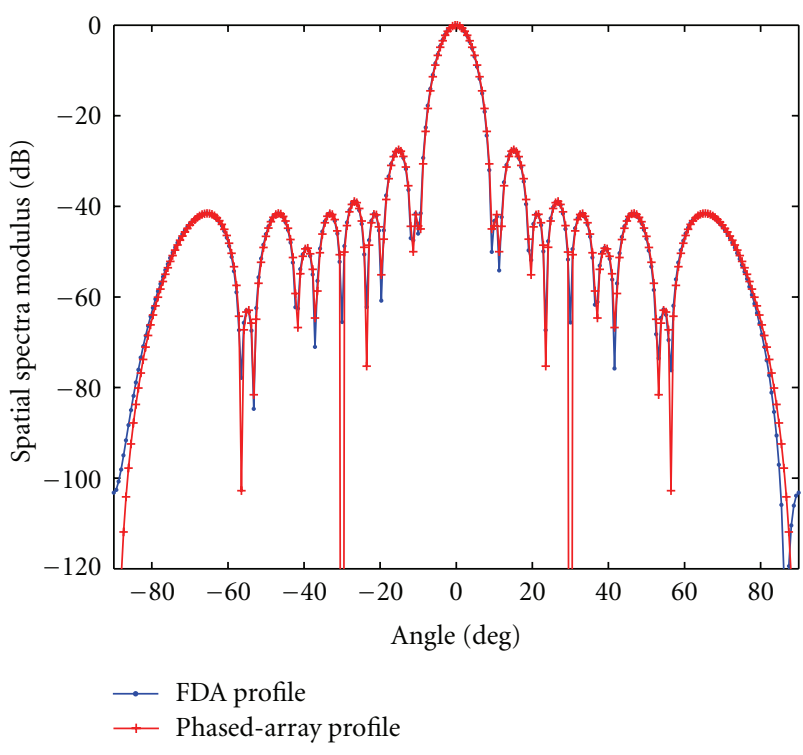

Figure 7: The comparative range profiles at the target's range.

phased arrays. It can be noticed that the FDA has a rangedependent beampattern which provides local maxima at different slant ranges. In contrast, the conventional phased array has a range-independent beampattern. The rangedependent beampattern offers a potential to suppress rangedependent interferences. Further, suppose that the target of interest is located at an angle $\theta_{0}=90^{\circ}$ and range $r=10 \mathrm{~km}$. Figure 6 shows the overall transmit/receive when compared to a phased array. It can also be noticed that, in this case, the FDA still has a range-dependent beampattern, whereas the phased array has a range-independent beampattern.

Example 2 (one target and two interferences with the same angle but different range). In this example, one target of interest is assumed to reflect a plane wave that impinges on the array from direction angle of $\theta_{0}=0^{\circ}$ and slant range of $r_{0}=10 \mathrm{~km}$. Further, suppose there are two interferences located as direction angle of $\theta_{1}=\theta_{2}=0^{\circ}$ and slant ranges of $r_{1}=8 \mathrm{~km}$ and $r_{2}=11 \mathrm{~km}$. The target power is fixed to $0 \mathrm{~dB}$, while the interference power is fixed to $40 \mathrm{~dB}$ and SNR is fixed to $10 \mathrm{~dB}$. Figure 7 shows the overall transmit/receive beamspace profile cut at the target's range. It can be noticed that the FDA radar and conventional phased-array radar have the same overall transmit/receive beamspace profile in the angle dimension. However, they have different beamspace profile in the range dimension as shown in Figure 8. The conventional phased-array radar has no resolution capability in the range dimension. In contrast, the FDA radar has a resolution capability in the range dimension. From (10), we know that the corresponding range resolution is determined by [35]

$$
\rho_{r}=\frac{c_{0}}{2 \cdot M \cdot \Delta f} .
$$

This resolution can efficiently suppress the range-dependent interferences which cannot be accessible for conventional phased-array radar.

Example 3 (one target and two interferences with different angle but different range). In this example, one target of interest is assumed to reflect a plane wave that impinges on the array from direction angle of $\theta_{0}=0^{\circ}$ and slant range of $r_{0}=10 \mathrm{~km}$. Further, suppose that there are two interferences located as direction angle of $\theta_{1}=0^{\circ}$ and $\theta_{2}=$ $25^{\circ}$ and slant ranges of $r_{1}=9 \mathrm{~km}$ and $r_{2}=1 \mathrm{~km}$. The target power is fixed to $0 \mathrm{~dB}$, while the interference power is fixed to $30 \mathrm{~dB}$ and SNR is fixed to $10 \mathrm{~dB}$. Figure 9 shows the overall transmit/receive beamspace profile cut at the target's range. It can be observed from this figure that the FDA exhibits a null at the location of the powerful interference located at $\theta=25^{\circ}$. Figure 10 shows the overall transmit/receive beamspace profile cut at the target's angle. It can be observed from this figure that the FDA exhibits a null at the location of the powerful interference located 


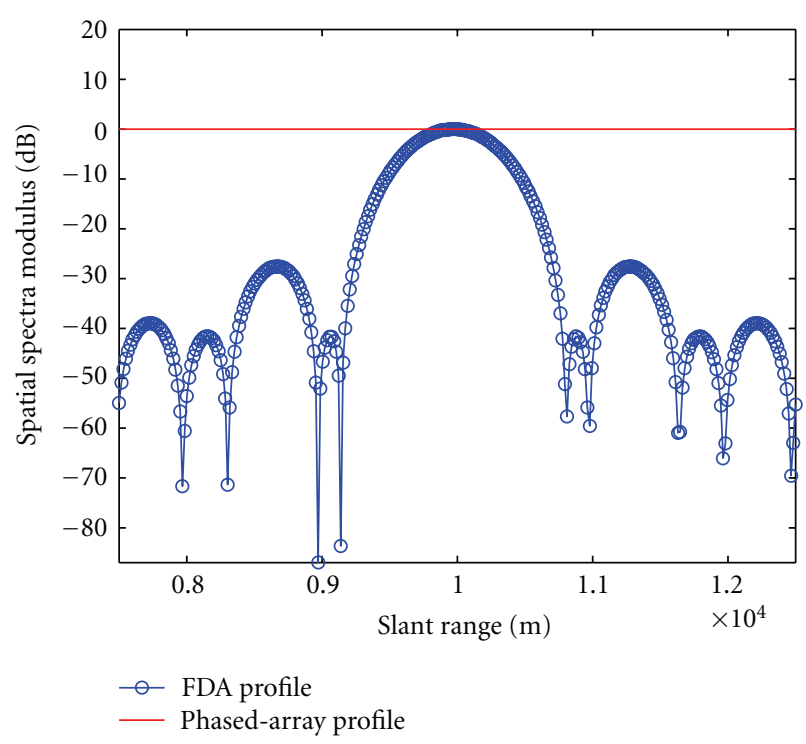

Figure 8: The comparative angle profiles at the target's angle.

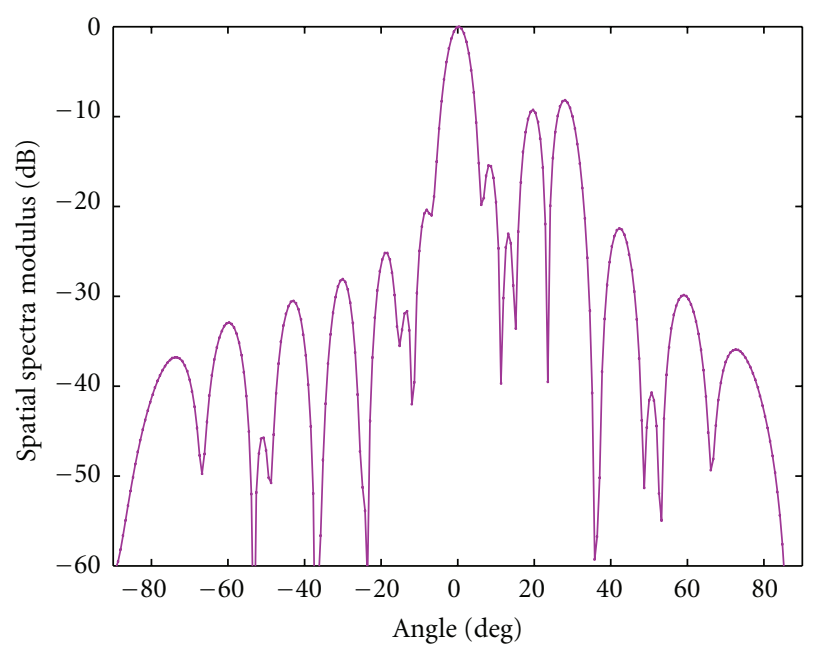

FIGURE 9: The range profiles of the transmit/receive beamforming output at the target's range.

at $r=9 \mathrm{~km}$. Therefore, the FDA enjoys the advantages of range-dependent beamforming capability, which provides a potential to suppress range-dependent interferences.

Example 4 (SINR analysis). In this example, we simulated the SINR performance. The output SINR performance is examined by the nonadaptive conventional beamformer. Figure 11 shows the output SINR versus SNR for the FDA and phased-array radars, where the interference-plus-noise ratio (INR) is fixed to $30 \mathrm{~dB}$. It can be noticed that the output SINR for the FDA radar has a much higher SINR as compared to the phased-array radar. This observation agrees with the fact that the FDA radar has more interference and noise robust than the phased-array radar.

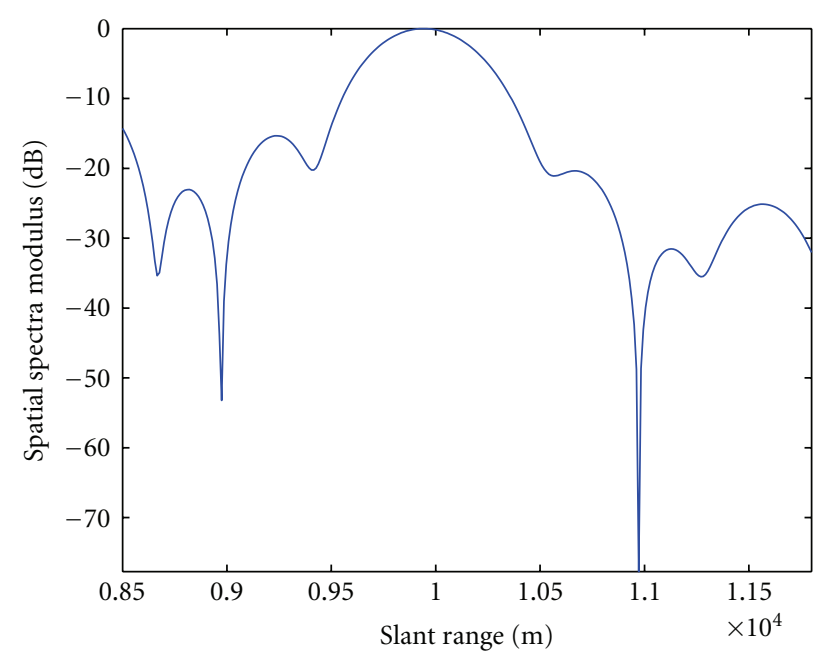

FIGURE 10: The angle profiles of the transmit/receive beamforming output at the target's angle.

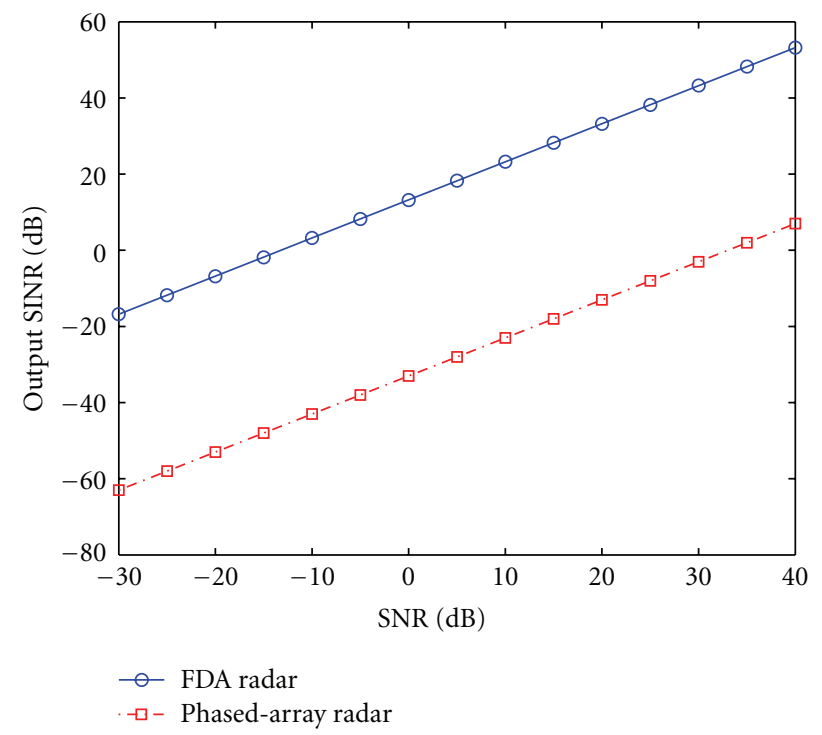

FIgURE 11: The comparative output SINR versus SNR at a fixed $\mathrm{INR}=30 \mathrm{~dB}$.

\section{Conclusion}

FDA has received much attention in recent years; however, little work about the range-angle-dependent beamforming and the corresponding applications has been published. This paper proposes a range-angle-dependent beamforming for FDA radars. The use of frequency increment generates an antenna pattern that is a function of range, time, and angle. The range-angle-dependent beamforming allows the FDA radar to transmit energy over a desired range or angle. This provides a potential to suppress range-dependent clutter and interference which is not accessible for conventional phasedarray radars. In this paper, the FDA radar signal model is formed and the range-angle-dependent beamforming is presented. The beamforming performance is examined by 
analyzing the transmit/receive beampatterns and the output SINR performance. Numerical simulation examples and discussions are also provided. The results show that the FDA radar indeed provides a significantly SINR performance improvement when compared to the conventional phasedarray radar. More importantly, the FDA radar provides a range-angle-dependent beampattern which provides promising potentials for suppressing range-angle-dependent interferences and many other advantages. The applications of the range-angle dependent beamforming in modern radars will be further investigated in our subsequent work.

\section{Acknowledgments}

This work was supported in part by the National Natural Science Foundation of China under Grant no. 41101317, the Fundamental Research Funds for the Central Universities under Grant no. ZYGX2010J001, the First Grade of 49th Chinese Post-Doctoral Research Funds under Grant no. 20110490143, and the Open Funds of the State Laboratory of Remote Sensing Science, Institute of Remote Sensing Applications, Chinese Academy of Sciences under Grant no. OFSLRSS201011.

\section{References}

[1] M. Bachmann, M. Schwerdt, and B. Bräutigam, "Accurate antenna pattern modeling for phased array antennas in SAR applicationsłdemonstration on TerraSAR-X," International Journal of Antennas and Propagation, vol. 2009, Article ID 492505, 9 pages, 2009.

[2] M. Elmer, B. D. Jeffs, K. F. Warnick, J. R. Fisher, and R. D. Norrod, "Beamformer design methods for radio astronomical phased array feeds," IEEE Transactions on Antennas and Propagation, vol. 60, no. 2, part 2, Article ID 6058599, pp. 903914, 2012.

[3] T. A. Lam, D. C. Vier, J. A. Nielsen, C. G. Parazzoli, and M. H. Tanielian, "Steering phased array antenna beams to the horizon using a buckyball NIM lens," Proceedings of the IEEE, vol. 99, no. 10, pp. 1755-1767, 2011.

[4] J. Landon, B. D. Jeffs, and K. F. Warnick, "Model-based subspace projection beamforming for deep interference nulling," IEEE Transactions on Signal Processing, vol. 60, no. 3, Article ID 6093978, pp. 1215-1228, 2012.

[5] M. Ettorre, R. Sauleau, and L. Le Coq, "Multi-beam multilayer leaky-wave SIW pillbox antenna for millimeter-wave applications," IEEE Transactions on Antennas and Propagation, vol. 59, no. 4, pp. 1093-1100, 2011.

[6] L. Manica, P. Rocca, G. Oliveri, and A. Massa, "Synthesis of multi-beam sub-arrayed antennas through an excitation matching strategy," IEEE Transactions on Antennas and Propagation, vol. 59, no. 2, pp. 482-492, 2011.

[7] M. Comisso and R. Vescovo, "Multi-beam synthesis with null constraints by phase control for antenna arrays of arbitrary geometry," Electronics Letters, vol. 43, no. 7, pp. 374-375, 2007.

[8] P. Antonik, M. C. Wicks, H. D. Griffiths, and C. J. Baker, "Frequency diverse array radars," in Proceedings of the IEEE Radar Conference, pp. 215-217, Verona, NY, USA, April 2006.

[9] W. Q. Wang, "Space-time coding MIMO-OFDM SAR for high-resolution imaging," IEEE Transactions on Geoscience and Remote Sensing, vol. 49, no. 8, pp. 3094-3104, 2011.
[10] W. Q. Wang and J. Y. Cai, "MIMO SAR using chirp diverse waveforms for wide-swath remote sensing," IEEE Transactions on Aerospace and Electronic Systems, vol. 48, no. 4, pp. 1-15, 2012.

[11] W.-Q. Wang, "Virtual antenna array analysis for MIMO synthetic aperture radars," International Journal of Antennas and Propagation, vol. 2012, Article ID 587276, 10 pages, 2012.

[12] K. V. Shanbhag, A. Deb, and M. Kulkarni, "MIMO radar with spatial-frequency diversity for improved detection performance," in Proceedings of the IEEE International Conference on Communication Control and Computing Technologies (ICCCCT'10), pp. 66-70, Nagercoil, India, October 2010.

[13] R. S. Adve, L. Applebaum, M. C. Wicks, and R. A. Schneible, "Space-time-waveform adaptive processing for frequency diverse distributed radar apertures," in Proceedings of the 40th Annual Conference on Information Sciences and Systems (CISS '06), pp. 1413-1417, March 2006.

[14] P. Antonik, M. C. Wicks, H. D. Griffiths, and C. J. Baker, "Multi-mission multi-mode waveform diversity," in Proceedings of the IEEE Radar Conference, pp. 580-582, April 2006.

[15] M. Secmen, S. Demir, A. Hizal, and T. Eker, "Frequency diverse array antenna with periodic time modulated pattern in range and angle," in Proceedings of the IEEE Radar Conference, pp. 427-430, Boston, Mass, USA, April 2007.

[16] S. Huang, K. F. Tong, and C. J. Baker, "Frequency diverse array with beam scanning feature," in Proceedings of the IEEE Antennas and Propagation Conference, San Diego, Calif, USA, July 2008.

[17] L. Zhuang, X. Liu, and W. Yu, "Precisely beam steering for frequency diverse arrays based on frequency offset selection," in Proceedings of the International Radar Conference (RADAR '09), pp. 1-4, Bordeaux, France, December 2009.

[18] P. Antonik, M. C. Wicks, H. D. Griffiths, and C. J. Baker, "Multi-mission multi-mode waveform diversity," in Proceedings of the IEEE Radar Conference, pp. 580-582, Verona, NY, USA, April 2006.

[19] M. C. Wicks and P. Antonik, "Frequency diverse array with independent modulation of frequency, amplitude, and phase, U.S.A," Patent 7, 319, 427, January 2008.

[20] M. C. Wicks and P. Antonik, "Method and apparatus for a frequency diverse array," U.S.A Patent 7. 511, 665B2, March 2009.

[21] M. Secmen, S. Demir, A. Hizal, and T. Eker, "Frequency diverse array antenna with periodic time modulated pattern in range and angle," in Proceedings of the IEEE Radar Conference, pp. 427-430, Boston, Mass, USA, April 2007.

[22] J. Huang, C. Baker, and K. F. Tong, "Frequency diverse array: simulation and design," in Loughborough Antennas and Propagation Conference, LAPC 2009, pp. 253-256, Loughborough, UK, November 2009.

[23] J. Farooq, M. A. Temple, and M. A. Saville, "Application of frequency diverse arrays to synthetic aperture radar imaging," in Proceedings of the International Conference on Electromagnetics in Advanced Applications (ICEAA '07), pp. 447-449, September 2007.

[24] J. Farooq, M. A. Temple, and M. A. Saville, "Exploiting frequency diverse array processing to improve SAR image resolution," in Proceedings of the IEEE Radar Conference (RADAR '08), pp. 1-5, Rome, Italy, May 2008.

[25] P. Baizert, T. B. Hale, M. A. Temple, and M. C. Wicks, "Forward-looking radar GMTI benefits using a linear frequency diverse array," Electronics Letters, vol. 42, no. 22, pp. 13111312, 2006. 
[26] P. F. Sammartino and C. J. Baker, "The frequency diverse bistatic system," in Proceedings of the International Waveform Diversity and Design Conference (WDD '09), pp. 155-159, Orlando, Fla, USA, February 2009.

[27] P. F. Sammartino and C. J. Baker, "Developments in the frequency diverse bistatic system," in Proceedings of the IEEE Radar Conference (RADAR '09), pp. 1-5, May 2009.

[28] P. F. Sammartino, C. J. Baker, and H. D. Griffiths, "Rangeangle dependent waveform," in Proceedings of the IEEE International Radar Conference (RADAR '10), pp. 511-515, May 2010.

[29] Y. G. Chen, Y. T. Li, Y. H. Wu, and H. Chen, "Research on the linear frequency diverse array performance," in Proceedings of the IEEE 10th International Conference on Signal Processing (ICSP '10), pp. 2324-2327, Beijing, China, October 2010.

[30] T. Higgins and S. D. Blunt, "Analysis of range-angle coupled beamforming with frequency-diverse chirps," in Proceedings of the International Waveform Diversity and Design Conference (WDD '09), pp. 140-144, Orlando, Fla, USA, February 2009.

[31] W. Q. Wang and H. Z. Shao, "A flexible phased-MIMO array antenna with beamforming on receive," International Journal of Antennas and Propagation, vol. 2012, Article ID 609598, 10 pages, 2012.

[32] B. W. Jung, R. S. Adve, J. Chun, and M. C. Wicks, "Detection performance using frequency diversity with distributed sensors," IEEE Transactions on Aerospace and Electronic Systems, vol. 47, no. 3, pp. 1800-1813, 2011.

[33] J. J. Zhang and A. Papandreou-Suppappola, "MIMO radar with frequency diversity," in Proceedings of the International Waveform Diversity and Design Conference (WDD '09), pp. 208-212, Kissimmee, Fla, USA, February 2009.

[34] H. L. Van Trees, Optimum Array Processing, Wiley, New York, NY, USA, 2002.

[35] J. R. Guerci, Space-Time Adaptive Processing for Radar, Artech House, Norwood, Mass, USA, 2003. 

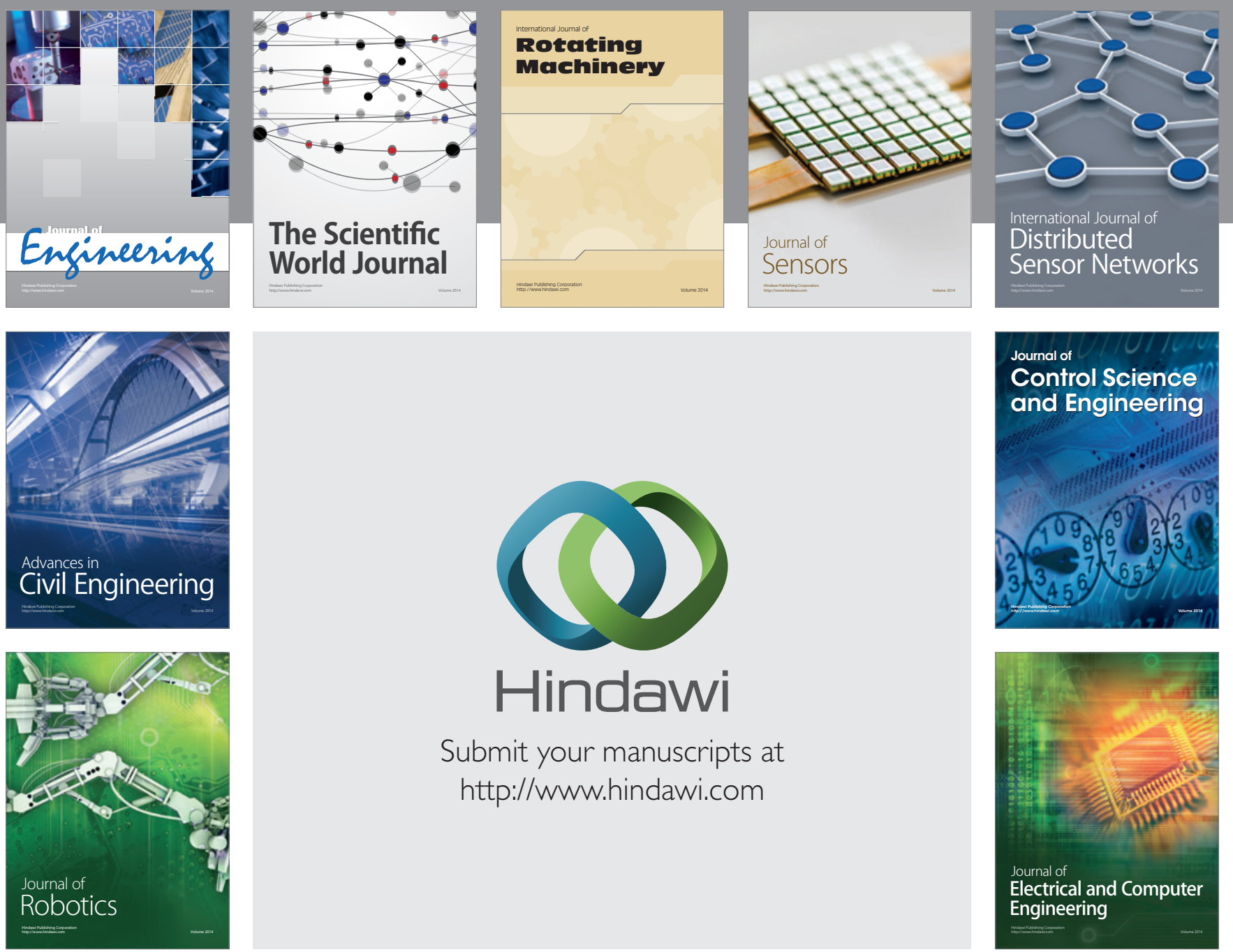

Submit your manuscripts at

http://www.hindawi.com
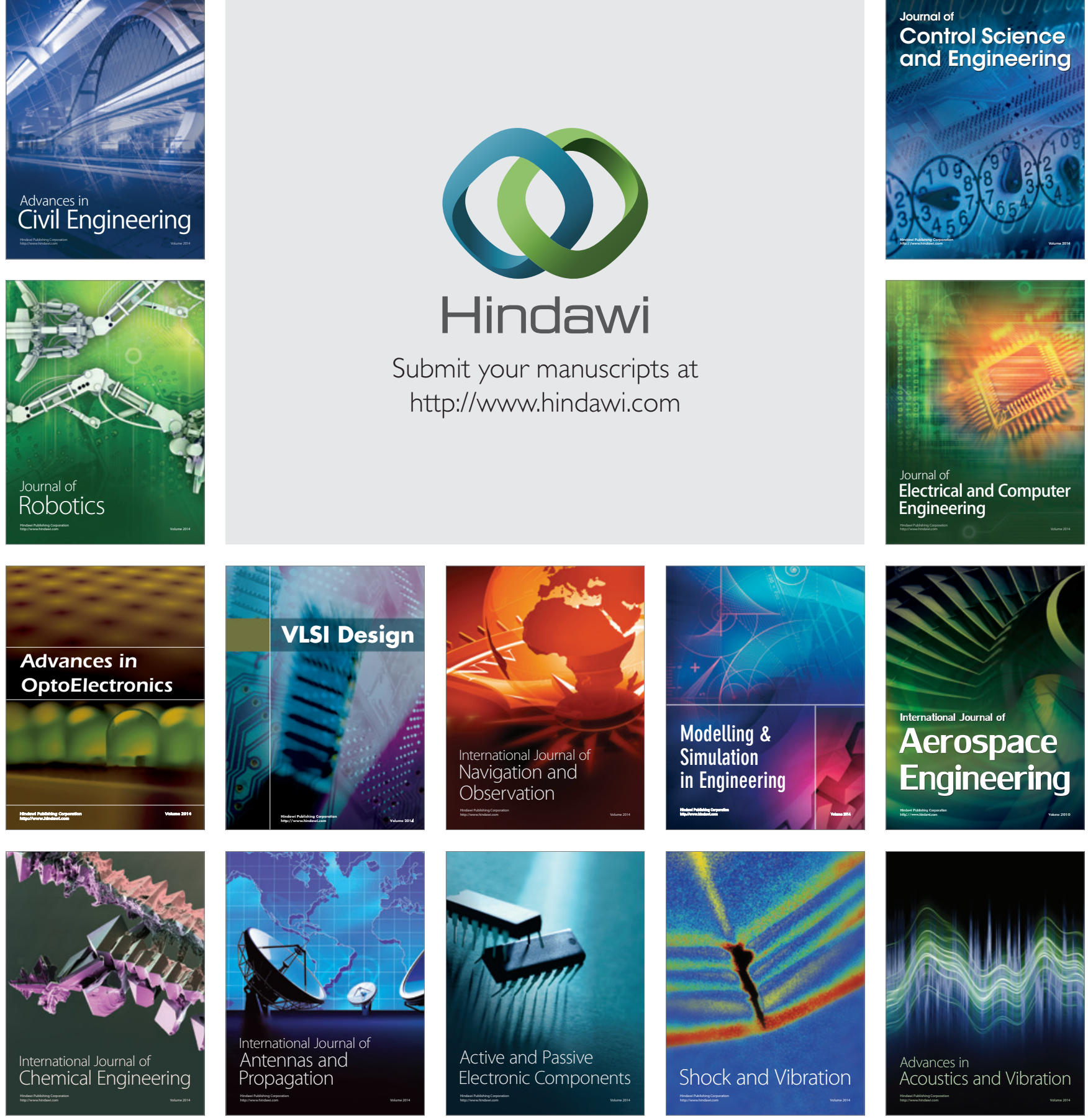